\title{
Doenças transmitidas por alimentos e participação da manipulação inadequada para sua ocorrência: uma revisão
}

\author{
Food borne diseases and participation of \\ inappropriate handling for their occurrence: a review
}

\author{
Larissa Soragni ${ }^{1}$ \\ Anderson Sena Barnabe ${ }^{2}$ \\ Tatiana Ribeiro de Campos Mello ${ }^{3}$
}

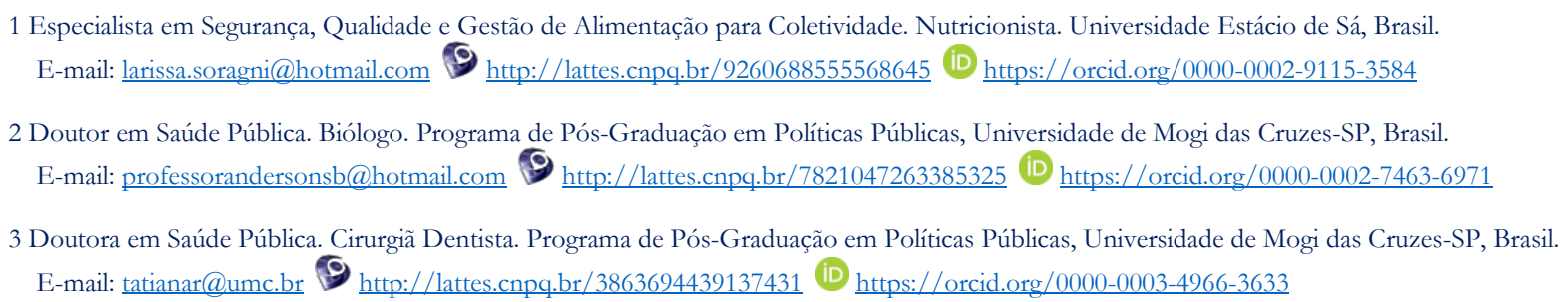

RESUMO: Introdução: A segurança alimentar está relacionada à inocuidade dos alimentos, com isso, estudos críticos e que disseminem dados para redução de doenças transmitidas por alimentos (DTAs) são necessários à prevenção dessas patologias, que afetam a população de modo global levando até mesmo a óbito. As DTAs são ocasionadas por água ou alimentos contaminados ocorrendo nos domicílios, tanto por quem os prepara, pelo ambiente e pelos procedimentos de higiene que nem sempre são eficazes. Objetivo: o objetivo deste trabalho é revisar a literatura acerca da manipulação de alimentos em domicílio e seu risco para desenvolvimento de DTAs. Método: revisão de artigos acerca do assunto, usando ferramentas computacionais. Resultados: foram encontrados 51 artigos sobre o tema, mas incluídos 12 desses, sendo os demais descartados por não se adequarem às exigências propostas. Conclusão: a residência desponta como principal local de ocorrência de DTAs, sendo as mais comuns causadas por Salmonella spp. e Staphylococcus aureus e apesar da importância das DTAs poucos são os estudos sobre o assunto.

Palavras-chave: Segurança alimentar. Contaminação de alimentos. DTAs. Domicílio.

\begin{abstract}
Introduction: Food safety is related to innocuity of foods, therefore, critical studies and dissemination of important data to reduce food borne disease (FBD) are necessary to prevent these diseases, which affect the population in a global way leading to death. FBDs are caused by contaminated water or food and there is a risk of occurrence in home, by the person who prepares them, by the environment and by procedures that are not always effective. Objective: The goal of this work is to review the literature on the manipulation of food at home and its risk for the development of FBDs. Method: Review articles about the subject. Results: 51 articles were found on the topic but only 12 of them were used, the others were discarded because they didn't fit the proposed requirements. Conclusion: the residence appears as the main place of occurrence of FBDs, being the most common caused by Salmonella spp. and Staphylococcus aureus, and despite the importance of FBDs there are few studies on the subject.
\end{abstract}

Keywords: Food safety. Food contamination. FBDs. Residence.

\section{INTRODUÇÃO}

O conceito de segurança alimentar se divi- de em duas vertentes, sendo no contexto sanitário resultado da inocuidade, integridade e autenticidade; e na vertente nutricional garan- 
tindo com acesso contínuo em quantidades suficientes de alimentos sem comprometer acesso a outras necessidades básicas, buscando a promoção a saúde e respeitando a diversidade (LEIT; WAISSMANN; VEGII, 2007)

A Organização Mundial da Saúde (OMS) (apud BOULOS, 1999, p. 21-23) preconiza que "a alimentação deve ser disponível em quantidade e qualidade nutricionalmente adequadas, além de ser livre de contaminações que possam levar ao desenvolvimento de doenças de origem alimentar" com isso qualquer risco de contaminação microbiológica dos alimentos apresenta um risco a saúde e segurança alimentar com a possibilidade de gerar uma enfermidade (MESQUITA et al, 2006.).

Doenças transmitidas por alimentos (DTAs) são causadas e disseminadas no mundo todo devido a contaminação microbiana ou parasitária de água e alimentos de origem vegetal ou animal.

Diversos são os agentes microbianos causadores de DTAs que, somados a fatores como a exposição a água contaminada e más condições de higiene, geram riscos para a ocorrência de patologias tanto na forma de infecções ou intoxicações: acometendo de forma crônica ou aguda; podendo caracterizar surtos epidêmicos; sendo disseminada ou localizada e se apresentando de variadas formas clínicas (BRASIL, 2010). As DTAs são parte de um dos maiores problemas de saúde pública, atingem toda população e majoritariamente as camadas menos favorecidas, crianças e idosos (WELKER et al., 2010). É importante ressaltar que além dos riscos fisiopatológicos, essas doenças implicam em, por exemplo: oneração ao sistema de saúde pública. Dados do Estado do Paraná, do ano de 2000, estimam gasto médio por paciente de $\mathrm{R} \$$ 1.870,00. Extrapolando esse valor para números nacionais de DTAs, R\$1.870.000,00 podem ter sido gastos com internações somente relacionadas a essas doenças (VAN AMSON; HARACEMIV; MASSON, 2006).
A saúde e a alimentação estão relacionados às práticas cotidianas e por consequência, ao desenvolvimento tecnológico e alteração de padrão do consumo, o que culminou na mudança dos padrões sanitários de toda a cadeia de produção, aumentando assim a necessidade de minimizar os riscos de contaminação e disseminação das doenças transmitidas por alimentos (SANTOS et al., 2011). A ingestão de alimentos que não atendem aos padrões sanitários — seja por representarem perigos físicos, químicos e, principalmente, biológicos - são um risco iminente à segurança alimentar (TONDO; BARTZ, 2014), sendo a contaminação por agentes biológicos a maior causadora dessas doenças (VAN AMSON; HARACEMIV; MASSON, 2006). Segundo Oliveira et. al. (2010), em artigo de revisão, poucos casos de DTAs são registrados oficialmente em sistemas de vigilância a saúde, sendo subnotificados praticamente em todo o mundo, e mesmo com essa falta de notificação os números de DTAs continuam aumentando, porém, sendo notificadas geralmente surtos que envolvem maior número de pessoas ou ainda sintomas mais graves e prolongados, evidenciando a necessidade de maiores informações sobre casos e formas de prevenção para sua redução.

Muitos dos alimentos contaminados aparentam características sensoriais normais sem alterações de textura, sabor e odor dessa forma muitas vezes quem o consome não tem percepção e consciência de que o alimento que parece perfeito ao olhar traz, intrinsicamente, risco ao ser ingerido podendo culminar em DTAs (MARCHI et al., 2012). Essa dificuldade em avaliar a presença de micro-organismos patogênicos se dá pela dose infectante de patógenos alimentares: normalmente é menor que a quantidade de microorganismos essencial para degradar os alimentos e dificultar que se consiga identificar alterações organolépticas como fontes de contaminação, assim sendo difícil rastrear os ali- 
mentos causadores de surtos e seu agente causador (DE OLIVEIRA et al, 2010).

Existem aproximadamente 250 tipos de doenças alimentares causadoras de síndromes diarreicas e diarreias sanguinolentas; quadros de maior gravidade como o de síndromes neurológicas, ictéricas, renais, alérgicas, respiratórias e septicêmicas (SÃO PAULO, 2008)sendo que muitas são causadas por micro-organismos patogênicos causadores de graves problemas de saúde pública e importantes perdas econômicas (VAN AMSON; HARACEMIV; MASSON, 2006). Dentre as DTAs, os agentes mais comuns no Brasil entre os anos 2000 e 2015 foram Salmonella spp; Staphbylococcus aureus; Escherichia coli e Bacilos cereus (BRASIL, 2015). A Salmonella spp é um dos principais agentes etiológicos sendo uma bactéria gram negativa entérica, geradora de infecções alimentares graves (GAUCI; GAUCI, 2005), com período de incubação entre 12 e 72 horas, durando entre 2-7 dias tendo como principais sintomas a diarreia e vômito. O Staphbylococcus aureus é gerador de intoxicação alimentar, com toxina resistente a pasteurização e fervura, período de incubação é de 1 a 6 horas, com duração de 1-2 dias, sendo o vômito seu principal sintoma. Escherichia coli é uma bactéria gram negativa, causadora tanto de infecção quanto toxinfecção alimentar, seu período de incubação é de 12 a 72 horas e a doença dura entre 2-7 dias e seus principais sintomas são diarreia e vômito. Bacilos cereus é uma bactéria gram positiva, a DTA dura entre 1-2 dias, os principais sintomas são diarreia e vômito dependendo da toxina, com período de incubação diferente para vômito (1-6 horas) e diarreia (8-24 horas), essa bactéria é formadora de endósporo, sendo tanto este como a toxina emética resistente à fervura e pasteurização, já a toxina diarreica não (TONDO;BARTZ, 2014).

Segundo dados do Ministério da Saúde, até outubro de 2015, dos 426 casos de surto de DTAs 58,5\% não tiveram agentes etiológicos identificados; 51\% dos alimentos causadores não foram identificados e, entre 2000 e 2015, $38,4 \%$ ocorreram nas residências, levando até mesmo a óbito (BRASIL, 2015), ficando clara a necessidade de se investigar a fonte e forma de transmissão, reduzindo riscos de disseminação da doença, redução da gravidade do problema e agravos à saúde e fomentar medidas de controle e prevenção de futuros surtos (BRASIL, 2015). Se comparados a dados anteriores, os casos de DTAs no Brasil diminuíram de 2000 a 2015, porém mesmo com redução de surtos a virulência continua equiparada a anos em que houve o dobro de surtos. Em comparação direta de dados até outubro de 2015 com 2014 houve redução em quase $50 \%$ dos casos de DTAs, passando de 886 casos; 15.700 doentes; 124.359 expostos; 9 mortos e virulência de 0,06\% em 2014 para 426 casos; 7.371 doentes; 18.766 expostos; 4 mortos e virulência de 0,05\% em 2015.

As DTAs originadas no lar, provavelmente, ocorrem como consequência de falhas nas condições de higiene e na segurança ao lidar com o alimento (GAUCI; GAUCI, 2005) como: conservação ineficiente dos alimentos; falhas nas operações de cocção e contaminação cruzada, possível também de acontecer em cozinhas domiciliares e não somente em cozinhas profissionais (ETCHEPARE et al., 2011), evidências indicam que parcelas importantes desses aconteçam em casa, tanto pelos alimentos como pela água, por falhas nesses procedimentos (REDMOND; GRIFFITH, 2003). Na manipulação doméstica cinco práticas representam maiores riscos à segurança alimentar, sendo elas: contaminação cruzada, e nesse risco pode se incluir tanto o contato com manipuladores, local em que se lida com o alimento e utensílios usados no preparo das refeições (GREIG; RAVEL, 2009); baixa higiene pessoal e ambiente impróprio para manipulação; ineficiência no controle de temperatura e consumo de alimentos que apresentem perigo à saúde ( GAUCI; GAUCI, 2005). 
Com a presença permanente de DTAs ligadas ao consumo alimentar domiciliar alguns estudos têm qualificado e quantificado o conhecimento da população sobre as doenças transmitidas por água e alimentos e seu impacto na saúde (ANGELILLO et al., 2001; MEER; MISNER, 2000). Com todas as informações sobre riscos da manipulação indevida, o presente estudo busca avaliar a literatura, ampliando a análise via ferramentas computacionais (Publish or Perish) sobre tema e compilar informações sobre o preparo de alimentos, evitando pontos comuns de contaminação que podem culminar para as DTAs.

\section{MÉTODO}

O presente estudo possui caráter exploratório, com metodologia de revisão de literatura. Foi delimitado primeiramente pelo contexto de segurança alimentar de forma geral, posteriormente aprofundando-se em conceitos e dados sobre DTAs e sua importância no contexto geral, por fim voltado para o preparo e manipulação de alimentos em domicílio, visto que não há treinamento para manipuladores do lar.

A pesquisa foi realizada em periódicos, jornais e revistas em português, inglês e espanhol. A pesquisa foi realizada de setembro de 2016 a janeiro de 2017, valendo-se da ferramenta computacional Publish or Perish (www.harzing.com), que se vale do buscador Google Scholar para busca em bases de dados a partir dos critérios utilizados, sendo verificado os coeficientes científicos dos artigos pela avaliação dos fatores de impacto e índice h.

Os critérios de inclusão de artigos foram artigos científicos sobre segurança alimentar; artigos que demonstrassem o impacto das DTAs e artigos sobre risco para saúde e sobre perigos de DTAs pela manipulação de alimentos no lar. Foram excluídos materiais informativos e artigos que não abrangessem o tema do presente estudo e com índice h menor que
20 citações. Após seleção de artigos e separação por temas foi realizada a revisão literária.

\section{RESULTADOS}

A revisão de literatura foi finalizada em 17 de janeiro de 2017. Ao todo foram encontrados 51 artigos sobre o tema, dos quais após leitura cautelosa apenas 12 artigos foram selecionados. Os demais artigos não foram incluídos por não abordarem o tema por completo, ignorando a ocorrência de surtos em domicílio ou por apresentarem índice $h$ menor que o estipulado como corte (4).

\section{REVISÃO DE LITERATURA}

Nadvorny, Figueiredo e Schimdt (2004) analisaram a ocorrência de surtos de intoxicação alimentar no Rio Grande do Sul a partir da coleta de dados disponibilizados pela Secretaria de Saúde do Rio Grande do Sul, encontrando 99 relatórios, sendo 74 causados por Salmonella sp. causada principalmente por consumo de alimentos preparados com ovos $(72,2 \%)$ - com base em anamnese — , sendo que $48,6 \%$ desses casos se originaram em domicílio. O estudo concluiu que a Salmonella sp. é o mais comum em surtos alimentares, tendo seu início na cadeia avícola, gerando matéria-prima de má qualidade que juntamente à manipulação inadequada levou a ocorrência desses surtos.

Estudo de Oliveira et al. (2010), aplicado a uma revisão da literatura mundial sobre DTAs, corroboram as informações de Nadvorny, Figueiredo e Schimdt (2004) e também apontaram Salmonella sp. como agente etiológico mais detectado em surtos, sendo o ovo o alimento causador de DTAs mais comum, sendo essa informação corroborada também pelo estudo de Kottowitz et al. (KOTTWITZ et al., 2009) que indica que 45\% dos casos estudados foram causados por ovos. Dados do Sistema de Vigilância Epidemiológica no Brasil, entre 1999 a 2008, indicam que do total 
de 6.062 surtos apenas 3.984 foram investigados e desses $23 \%$ tiveram ovo cru ou mal cozido como principal alimento causador de doenças. Quanto ao local de ocorrência de surtos, estabelecimentos comerciais e residências, respectivamente, foram os locais de maior incidência em todo mundo, sendo o Rio Grande do Sul contrário a essa ordem, tendo como principal local as residências. O estudo concluiu que ainda há pouca investigação de surtos alimentares, sendo os de maior virulência ou que afetaram mais pessoas os mais investigados, além disso a má manipulação, conservação e cocção são as maiores causas de DTAs. Quanto aos agentes causadores, a Salmonella sp., Staphylococcus aureus, Bacillus cereuse Escherichia coli aparecem como os mais comuns às DTAs e se suspeita que a Listeria monocytogenes seja a principal bactéria letal.

No trabalho de Welker et al. ( 2010), fora realizada análise microbiológica das sobras de alimentos envolvidos em surtos de DTAs no Rio Grande do Sul entre 2006 e 2007, sendo analisados no total 186 surtos — dos quais 104 eram positivos para os microorganismos estudados - e 704 amostras sendo em média 4 amostras para cada surto, resultando em $30 \%$ de detecção para algum microorganismo. Dos surtos analisados 44\% não teve determinação de agente causador por inúmeras causas prováveis, como inativação do agente etiológico por inadequação do armazenamento ou transporte; não uniformidade de microorganismos na amostra ou método indisponível no laboratório para sua determinação. Com relação aos resultados, tem-se que ao se considerar amostras com contaminação maior que $1,0 \times 10^{3} \mathrm{UFC} / \mathrm{g}$ ou NMP/g- ou para Salmonella $s p p$. presença em $25 \mathrm{~g}$ - os principais microorganismos identificados foram Salmonella spp. (37\%), Estafilococos coagulase positiva (28\%) e Escherichia coli (22\%). Quando se trata dos alimentos envolvidos, tem-se produtos cárneos - com 36\% de contaminação foram os mais comuns. Em se tratando do local de ocorrência de surtos desse estudo, as residências aparecem como principal local de ocorrência de DTAs com 43\% de incidência. Os resultados desse estudo apontam a necessidade de se focar na segurança dos alimentos e na necessidade de educar e orientar a população quanto a manipulação, conservação e boas práticas no domicílio para reduzir o número de DTAs.

Nos estudos de Baptista e Antunes (2005), se observou casos de surtos alimentares em Portugal por números de casos entre 1987 e 1998, havendo nesse primeiro ano 215 casos e no último 1411 casos, demonstrando grande aumento de incidência. Entre 1993 e 1998, fora recolhido pelo Instituto Nacional de Saúde da região do Porto dados quanto ao agente causador, sendo a maioria 44,1\% de agente desconhecido, apontando a necessidade de maior estudo sobre a microbiota causadora de DTAs. Outro ponto analisado foi o local de ocorrência desses surtos sendo domicílios com 28,8\% local de maior incidência, assim como estudos nacionais, como de Nadvorny, Figueiredo e Schimdt (2004) que apontaram as residências como local de maior incidência de DTAs. Quanto ao tipo de alimento consumido e causador de surtos, tem-se: bolos/ pastelarias / pré-misturas com 26,4\%. O trabalho aborda todos os aspectos para produção de alimentos seguros e aponta para sensibilização para a problemática das DTAs e como preveni-las, concluindo que a somatória de cuidados e atenção quanto a qualidade dos alimentos, adequação do local e manipulação correta culminam em um alimento seguro.

Estudo de Kottwitzet al. (2009), por meio de compilação de dados valendo-se de avaliação das "Fichas de Investigação Epidemiológica de surtos notificados pelas Regionais de Saúde dos Municípios paranaenses" e de "Fichas de surtos concluídos epidemiologicamente", mas sem realização da confirmação laboratorial de DTA. Foram notificados 286 casos de salmonelose no período entre 1999 e 2008 
no Estado do Paraná- Brasil, sendo desses 254 comprovados laboratorialmente, tanto por pesquisa de cepas no doente como dos alimentos envolvidos. Nesse período 5.641 tiveram contato com alimentos contaminados com Salmonella spp., sendo que 35,9\% manifestaram os sintomas da doença, sendo 16,3\% hospitalizadas, havendo um óbito. Quanto aos alimentos envolvidos, alimentos a bases de ovos, com 45\%, foram os mais presentes. Quanto ao local de ocorrência, o domicílio se apresenta como principal, com 49,2\%. Como conclusão deste trabalho tem-se que maior parte dos surtos no período são causados por produtos à base de ovos, preparados para reuniões familiares, sendo a cepa Enteriditis a mais comum, outro fato importante relatado pelos autores é que a manipulação inadequada é de grande contribuição para a ocorrência de surtos alimentares, por contaminação cruzada, sendo necessária maior atenção quanto à educação e orientação aos manipuladores, tanto de estabelecimentos quanto em residência.

Artigo de Van Amson, Haracemiv e Masson (2006) realizou levantamento de dados acerca de DTAs no Estado do Paraná, entre 1978 e 2000, sendo essas informações coletadas em boletins mensais vindos de municípios paranaenses. Foram relatados 1.195 surtos, sendo os agentes etiológicos mais comuns o Staphylococcus aureus em 41,2\% e Salmonella sp. em 33,8\% dos casos. O estudo não aprofunda em número quanto aos alimentos envolvidos, contudo destaca o ovo como maior alimento veiculador de salmonelose estando envolvido em maior parte dos surtos ocorridos. Outro destaque quanto aos alimentos envolvidos são os produtos de origem animal, por apresentarem microorganismos vivos mesmo após o abate que podem contaminar outros alimentos e o fato de esses produtos apresentarem ótimos meios de crescimento bacteriano pela alta atividade de água; nutrientes e baixa acidez. Outro ponto abordado é o local de ocorrência, indicando como maior incidência a residência, com 50,5\%, explicando essa ocorrência pela baixa iniciativa quanto à educação sobre segurança alimentar, sendo de desconhecimento de maior parte da população condições básicas de higiene para manipulação, armazenamento e perigos para surgimento de DTAs. O presente estudo indica um aumento significativo nos casos de DTAs, aumentando gastos públicos para internações e cuidados que poderiam ser evitados com medidas preventivas. O estudo conclui que há maior necessidade de atenção à segurança alimentar para que se reduza o número de DTAs e para que haja maior controle sobre esses números seria necessário que houvesse maior presença da Vigilância Sanitária e notificação de surtos, além de implementação de ações de orientação e educação quanto a manipulação de alimentos em domicílios.

Estudo de Peresi et. al. (1998) realizou análise de dados de surtos de DTAs decorrentes de Salmonella entereditis ocorridos entre julho de 1993 e junho de 1997 no noroeste do Estadode São Paulo. Ocorreram 23 casos, sendo a metodologia de pesquisa baseada na aplicação de questionário intentando obter informações do número de expostos e afetados; idade; período de incubação; sintomas e hospitalizações; e alimentos envolvidos. Para análise do alimento veiculador, houve coleta de 14 amostras e 3 ovos do mesmo lote, sendo que 9 surtos não dispunham de alimento para análise. Para tipagem de cepa realizou-se também a coprocultura, que consiste na análise de amostra fecal. Do total de 23 surtos, 19 foram analisados, que envolveram 906 casos gerando 295 internações. Um resultado interessante é que em todas as amostras de alimentos foram encontradas cepas de Salmonella sp. e que em $95,7 \%$ dos casos desta bactéria tiveram o ovo como seu percursor. O estudo conclui que com a alta presença de ovos em surtos de salmonelose é necessária maior atenção ao seu consumo, produção e armazenamento, por causar uma doença grave que atinge toda po- 
pulação. O estudo, apesar de antigo, vai ao encontro de muitos atuais, e apesar de apresentar dados de difícil entendimento ao primeiro olhar, contribui para visão da evolução das DTAs e seu impacto na saúde.

Em seu estudo, Wilcock et al. (2004) dissertaram sobre a relação do consumidor com o alimento e a necessidade de garantia de práticas seguras na manipulação de alimentos garantindo a segurança alimentar embasadas em normas sanitárias. Nessa revisão os autores pontuam que o risco microbiológico é potencialmente maior pela rápida proliferação e pequena dose infectante e com inúmeros casos de DTAs acontece um medo generalizado sobre novos patógenos, gerando falta de confiança na cadeia produtiva de alimentos, além de altos custos com tratamentos. Em números, na Inglaterra e País de Gales só em 1991 houveram 23 mil casos de salmonelose tendo custo estimado entre 40 e 50 milhões de libras, já no Canadá há estimativa de 2,2 milhões de casos de DTAs por ano com gasto anual de 1,3 bilhões de dólares, nos Estados Unidos há estimativa de 6,5 a 33 milhões de pessoas atingidas por DTAs, com custos superiores a 9 milhões de dólares para tratamentos e perda de produtividade, e apesar dos altos números, muitas DTAs não estão nas estatísticas por falta de tratamento, desconhecimento do alimento causador da doença e contaminação interpessoal. Apesar de diversas iniciativas para melhorar a segurança alimentar muitos ainda desconhecem o papel crucial das DTAs na saúde pública. Em sua avaliação das atitudes dos consumidores perante os alimentos, determina que há diferentes pesos quanto a diferentes questões, não estando demasiadamente preocupados em mudar atitudes para aumentar a segurança dos alimentos. Pesquisas de opinião nos Estados Unidos apontam que a maioria das pessoas consideravam a contaminação de alimentos o maior risco a segurança alimentar e boa parte pagaria mais caro por um alimento mais seguro. Quanto às práticas de segurança alimentar, o trabalho demonstra que fatores predominantemente responsáveis por DTAs na Inglaterra e no País de Gales entre 1992 a 1994 e 1995 e 1996 foram: armazenamento inapropriado, cozimento ineficaz ou reaquecimento e a contaminação cruzada, sendo que grande parte dos consumidores não sabem que mais da metade dos casos de DTAs acontecem nas residências, além de não saberem de riscos dessas contaminações. Com tantos pontos abordados, fica evidente que os saberes e atitudes dos consumidores quanto à segurança dos alimentos são dispares e não geram sempre comportamentos que assegurem a segurança dos alimentos consumidos. O estudo conclui que é indispensável o suporte de profissionais para informar os consumidores quanto às questões de segurança alimentar, recomendando que se aprenda e ensine mais sobre condutas e comportamentos de consumidores; criação de percepção sobre segurança e insegurança alimentar; promoção de credibilidade pública e fontes de informação honestas acerca do assunto; investimentos em educação sobre segurança alimentar; necessidade de familiarizar o público com assunto e trazer para o cotidiano suas implicações.

Estudos de Nauta et al. (2008), abordaram de forma multidisciplinar o comportamento quanto a higiene dos alimentos produzidos em domicílio focando na disseminação de conhecimentos acerca da manipulação segura para que esta seja aplicada nas cozinhas domiciliares, mudando primeiramente as atitudes para posteriormente criar novos hábitos e comportamento. O estudo envolveu 240 pessoas no teste inicial que foram aleatoriamente divididas e embasou-se em informações autorelatadas baseada na sensação de "nojo" quanto a transferência e sobrevivência dos microorganismos, sendo abordadas no estudo três diferentes vertentes. A primeira vertente focou na disseminação de informações importantes quanto a segurança na produção de 
alimentos e práticas domésticas higiênicas sem associa-las a imagens negativas; a segunda incluídas nas mensagens linguagem "hostil" ligadas a risco e imagens de pessoas bravas e não a auto-preservação inseridas em fundo vermelho; e na terceira o nojo foi ligado ao perigo para gerar comportamento de auto preservação quanto a contaminação e sua ligação a doenças, havendo ainda fotos relacionadas a doentes em fundo verde com desagradável sombreamento. Fora utilizado também versão sobre o mesmo tema sem motivadores emocionais como caso controle. Após o teste, os 3.422 participantes foram randomicamente alocados para uma das quatro versões disponíveis sendo questionados sobre intenção de produzir alimentos seguindo práticas de higiene e motivação para se comportarem de forma que evitasse a contaminação cruzada. Após a conclusão desse inquérito fora realizada análise microbiológica da preparação proposta com participantes do grupo controle, do grupo com informações de contaminação e grupo com essas informações e lembrete dessas junto à receita. O frango utilizado fora contaminado propositalmente com Lactobacillus casei por este ser um bom marcador de contaminação cruzada por Campylobacter jejuni. Como resultado, observou-se que informações contendo emoções de nojo foram mais eficazes para motivar atitudes de autopreservação que aquelas contendo emoções agressivas e ainda mais que aquelas sem emoção. Quanto a intenção de realizar de autopreservação as informações passadas com emoção ligada ao nojo foram quase iguais àquelas passadas sem emoção e maior que aquelas ligadas a raiva. Quanto à análise microbiológica de 82 saladas incluídas na pesquisa, todas poderiam ser consumidas, sem disparidades significantes entre elas. Por ter uma abordagem em diferentes aspectos, o estudo mostrou que as informações aliadas a ação quanto a segurança dos alimentos na preparação atinge melhor a mudança de comporta- mento que somente educação quanto a segurança alimentar.

A European Food Safety Authority and the European Centre for Disease Prevention and Control, em 2013, junto ao relatório a partir da compilação de dados pelo Programa de Doenças Alimentares e Hídricas e Zoonoses pelo European Centre for Disease Prevention and Control, apresentaram as DTAs e zoonoses mais comuns na zona do euro (EU). Na área da União Europeia (UE), em 2013, foram relatados 5.196 surtos de origem alimentar, sendo maior parte causadas pelas bactérias Salmonella e Campylobacter, contudo 28,9\% dos casos o agente etiológico não fora descoberto. Quanto aos alimentos causadores, há ligação com ovos e produtos derivados de ovos, seguidos de alimentos mistos e peixes e seus derivados. No ano de 2013,o Campylobacter manteve-se como maior causador de doenças gastrointestinais notificados na EU, como vem ocorrendo desde 2005, sendo o número confirmado de Campylobacter foi 214.779 sendo de baixa letalidade. Os alimentos fontes dos casos de forte evidência foram em ordem crescente de importância: leite e misto de alimentos, outras, mistas ou não especificada carne de aves e produtos derivados, frangos de corte de carnes e produtos derivados. Quanto aos casos de Salmonella, nesse mesmo período houve 82.694 casos confirmados, sendo as cepas mais comuns foram S. enteritidis $(39,5 \%)$ havendo redução de 4.720 casos e S. typhimurium $(20,2 \%)$, S. enteritidis, sendo o alimento mais comum ovos e produtos à base de ovos seguido de carne de frango. O trabalho detalha cada DTA e zoonose e compara detalhadamente casos de cada uma dessas doenças por país, trazendo um panorama de alguns países na Europa analisando localmente os casos, sendo a França, Grã Bretanha e Espanha, respectivamente, os países com mais evidências fortes de DTAs e como alimentos mais comuns indicam ovos e produtos a base de ovos, alimentos mistos e peixes e produtos 
a base peixe. Quanto ao local de contaminação, as residências lideram com $38,5 \%$. O estudo conclui que houve redução do número de casos de DTAs, porém os dados notificados não são equiparados nos países, e a disseminação de agentes causadores e alimentos não são muitas vezes reais (AUTHORITY, 2015)

Estudo de Di Pietro et al. (2004) verificou surtos de DTAs na província de Rio Negro, Argentina, no período de 1993 a 2001, coletando dados no Sistema de Vigilância Epidemiológica Nacional (Sim. Na. V.E.) que abrange notificações de casos isolados e também, posteriormente, notificação de focos de DTAs quando há casos em diferentes locais com mesma fonte de início. Na província de Rio Negro, desde 1983 há avaliação dos alimentos vendidos realizado pela Direção de Saúde Ambiental para capacitação e padronização de técnicas para investigação de DTAs e a partir de1993 esses dados são inseridos em bancos. A metodologia baseou-se na definição DTAs a partir dos sintomas, seguido de coleta de dados a partir da notificação obrigatória na Si.Na.V.E por médicos e hospitais ou até mesmo dos afetados. A padronização das técnicas resultou em procedimento padronizado iniciado por entrevista pessoas sem sintomas como controle, mas que tinham ligação com possíveis focos de infecção valendo-se de coleta de dados padronizada do consumido nos últimos três dias com detalhes de hora e forma de preparo; prazo para começo sintomas, e os principais, além de informações sobre outros possíveis afetados; realizada vistoria sanitária da possível núcleo da DTA e coleta de amostra para análise microbiológica; amostragem de casos para estudo bacteriológico por vômito ou fezes; validação das estatísticas de infecção quanto ao alimento envolvido, tempo médio e pontuação dos sintomas mais presentes; análise das variáveis envolvidas: local de ocorrência, local de fabricação dos alimentos possivelmente envolvidos, o princi- pal alimento provável, local de naturalidade dos alimentos, agente etiológico causador da DTA atestada por análise do agente etiológico tanto por exames nos doentes, manipuladores como superfície de contato dos alimentos e posterior criação de relatório final. Como resultados dessa pesquisa obteve-se que a Salmonella é o agente mais comum, seguido por Staphylococcus aureus e Trichinella spiralis e por último a Escherichia coli. Quanto a casos a Salmonella spp. também é a principal causadora de DTAs, sendo a cepa $S$. enteritidis associada a ovos uma das mais comuns causadoras de DTAs no mundo. Quanto aos alimentos envolvidos em surtos os principais são: cárneos, queijos, carnes e sanduíches, sobremesas e sorvete, sendo o mais importante nos casos de DTA o sorvete. Já quanto a origem desses envolvidos, a maioria dos surtos se iniciou em casa. O estudo discute e conclui diversos pontos importantes, como a participação dos médicos e hospitais dos setores público e privados colabora para melhor compilação de dados, mesmo que ainda sejam necessárias melhorias para essa comunicação; quanto a metodologia, são necessários ajustes para melhor análise microbiológica; mais atenção a pontos críticos para contaminação como o tempo de cocção, contaminação pós cocção, grande espaço de tempo entre produção e consumo, armazenamento inadequado dos alimentos, má manipulação de alimentos e falta de higiene do manipulador e ambiente além de água contaminada. É muito importante o foco na pesquisa para que haja dados fidedignos que gerem gerando mínimos equívocos pois a única forma de conhecer os dados sobre as DTAs são esses dados para que eficientemente se evite a disseminação do surto.

Estudo de Van Asselt et al.(2008) usou um modelo de contaminação cruzada caseira no preparo de frango para salada a partir da análise de tábuas de corte, mãos e facas. A metodologia baseou-se na criação de diferentes cenários que possibilitariam a contaminação 
cruzada nessa preparação repetidos pelo menos quatro vezes no teste antes da aplicação, os cenários criados foram: se mãos e utensílios foram lavados com ou sem sabão entre as atividades, não lavagem ou descontaminação entre o corte de frango e salada. Avaliaram-se as taxas de transferência a partir da contagem de Campylobater jejuni e Lactobacillus casei sobreviventes na salada após finalização do preparo. Os resultados foram avaliados a partir dos diferentes cenários de contaminação, desde isoladas ou combinação de mãos, tábua de corte e facas enumerando no final do preparo a contagem de bactérias, sendo 25 o limite. No melhor cenário de contaminação cruzada todos os valores foram abaixo do limite, comprovando que práticas de higiene reduzem carga microbiana, sendo que cometer um erro durante o preparo é tão ruim quanto realizar todo o processo sem práticas de higiene. Por ser uma análise baseada em um modelo real mostrando-se mais realista sobre as taxas de transferência de Lactobacillus casei e Campylobater jejuni, sendo esses bons marcadores de contaminação cruzada em domicílios.

\section{CONCLUSÃo}

As DTAs apesar de terem grande importância na saúde pública, gerar, ainda hoje, diversos óbitos e apresentar grande impacto econômico, implicam em poucos estudos de relevância epidemiológica. Muitos pontos dificultam que trabalhos sobre DTAs sejam realizados, como a subnotificação e subsequente falta de análise do local de ocorrência e alimento envolvido, gerando números irreais que não contemplam a quantidade de surtos alimentares principalmente no que infere a casos isolados, sendo importante que haja notificação por parte dos envolvidos e dos serviços de saúde. Pode-se concluir, com embasamento de literatura, que grande parte dos casos ocorrem nas residências e que poderiam ser evitadas com cuidados mínimos de higiene como higienização de mãos e utensílios, cuidados com temperatura de cozimento, cuidados com armazenamento e com a contaminação cruzada, sendo que ensinamentos simples quanto às boas práticas de manipulação evitariam grande parte desses casos. Assim como observado em Oliveira e Resende (2017) que revisaram a importância da ocorrência de parasitoses, corroboramos seu texto, que infere a importância da interação e cooperação de órgãos ligados às áreas da Saúde, Meio Ambiente, Vigilância Sanitária e Agropecuária a fim de prover maior controle de qualidade dos alimentos e outros insumos.

Pode-se concluir também que cuidados devem ser tomados quanto ao preparo de ovos e produtos produzidos com estes, pois grande parte das DTAs são geradas por mau cozimento gerando a mais comum dessas doenças: a salmonelose. A pasteurização do ovo para produção de alimentos além do cozimento completo de ovos e outros alimentos evitaria grande parte dos casos desta doença e da maioria das DTAs. A contaminação por Staphylococcus aureus também aparece como uma das mais comuns, podendo ser evitada por instruções básicas de higiene pessoal na manipulação de alimentos. Logo, com toda fundamentação, fica evidente que se a população tivesse acesso ao conhecimento teóricoprático de manuseio de alimentos e os aplicasse, os números e gastos com DTAs seriam radicalmente reduzidos e causariam menos danos à população e à economia.

\section{REFERÊNCIAS}

ANGELILO, I. F., et al. "Consumers and foodborne diseases: knowledge, attitudes and reported behavior in one region of Italy." International Journal of Food Microbiology, v. 64, n 1, p. 161-166, mar 2001. https://doi.org/ 10.1016/S0168-1605(00)00451-7

AUTHORITY, E. F. S. et al. The European Union summary report on trends and sources of zoonoses, zoonotic agents and food-borne 
outbreaks in 2013. EFSa Journal, v. 13, n 1, jan-jun 2015. https://doi.org/10.2903/i.efsa.2015.3991

BAPTISTA, P., ANTUNES, C. "Higiene e Segurança Alimentar na Restauração-Volume II-Avançado". Forvisão-Consultoria em formação integrada, SA. v. 300, 2005. [citado 22 de novembro de 2016].

BOULOS, M. E. M. S. Segurança alimentar: uma preocupação - questão de atualizar e viabilizar informação. Nutrição em Pauta, p. 21-23, nov./dez. de 1999.

BRASIL. Conselho Nacional de Segurança Alimentar e Nutricional. "Políticas Públicas para Garantia da Alimentação Adequada e Saudável: A união das agendas da Saúde e da Segurança Alimentar e Nutricional". 5 Conferência Nacional de Segurança Alimentar e Nutricional, Brasília 2015: [citado 20 de setembro de 2016]. Disponível em: http:// www4.planalto.gov.br/consea/eventos/confe rencias/5a-conferencia-nacional-de-

seguranca-alimentar-e-nutricional

BRASIL. Ministério da Saúde, Secretaria de Vigilância em Saúde, Departamento de Vigilância em Saúde, Departamento de Vigilância das Doenças Transmissíveis, Coordenação Geral de Doenças Transmissíveis, Unidade de vigilância das Doenças de Transmissão Hídrica e Alimentar. Doenças Transmitidas por Alimentos. Brasília, 2015.

BRASIL. Ministério da Saúde. Secretaria de Vigilância em Saúde. Departamento de Vigilância Epidemiológica. Manual integrado de vigilância, prevenção e controle de doenças transmitidas por alimentos. Ministério da Saúde, Secretaria de Vigilância em Saúde, Departamento de Vigilância Epidemiológica.

- Brasília: Editora do Ministério da Saúde, 2010. 158 p.: il. - (Série A. Normas e Manuais Técnicos)

DE OLIVEIRA, A. B. A. et al. Doenças Transmitidas por Alimentos: Principais Agentes Etiológicos, Alimentos Envolvidos e Fatores Predisponentes. Revista HCPA, v. 30, n 3, p. 279-285, out. 2010.
DI PIETRO, S. et al. Vigilância epidemiológica de enfermedades transmitidas por alimentos em la provincia de Río Negro, Argentina, 1993-2001. Medicina. Buenos Aires, v. 64, n 2, p. 120-124, abr.-jul. 2004

ETCHEPARE, M. A.; DEON, B. C.; HECKTHEUER, L. H.; SACCOL, S. Avaliação das boas práticas de higiene de esponjas, panos de prato e tábuas de core no controle de DTAs no município de Santa Maria- RS. Congresso de Ciência e Tecnologia da UTFPR Campus Dois Vizinhos (pp. 102105), 2011.

GAUCI, C.; GAUCI, A. A. What does the food handler in the home know about salmonellosis and food safety? The journal of the Royal Society for the Promotion of Health. Londres, v. 125, n 3, p. 136-142, jan. 2005. https://doi.org/10.1177/146642400512500318

GREIG, J. D.; RAVEL, A. Analysis of foodborne outbreak data reported internationally for source attribution. International journal of food microbiology, v. 130, n 2, p. 77-87, mar. 2009. https://doi.org/10.1016/j.ijfoodmicro.2008.12. $\underline{031}$

KOTTWITZ, L. B. M. et al. Avaliação epidemiológica de surtos de salmonelose ocorridos no período de 1999 a 2008 no Estado do Paraná, Brasil. Acta Scientiarum. Health Sciences. Maringá, v. 32, n 1, p. 9-15, jan-jun. 2009. https://doi.org/10.4025/actascihealthsci.v32i1.6340 LEITE, L. H. M.; WAISSMANN, W.; VEGGI, A. B. Reprodutibilidade de um questionário para avaliação de conhecimentos, percepções e práticas em segurança sanitária alimentar de portadores de HIV/AIDS ambulatoriais. Cad Saúde Pública. São Paulo, vol 4, n. 23, p. 971-6, abr. 2007. https://doi.org/10.1590/S0102311X2007000400024

MARCHI, D. M. et al. Ocorrência de surtos de doenças transmitidas por alimentos no Município de Chapecó, Estado de Santa Catarina, Brasil, no período de 1995 a 2007. Epidemiologia e Serviços de Saúde, Brasília, vol 21, n 3, p. 401-407, dez 2012. https://doi.org/ $\underline{10.5123 / \mathrm{S} 1679-49742011000300015}$ 
MEER, R. R.; MISNER, S. L. "Food safety knowledge and behavior of expanded food and nutrition education program participants in Arizona. Journal of Food Protection, Des Moines, vol 63, n 12, p. 1725-1731, fev. 2000. https://doi.org/10.4315/0362-028X-63.12.1725

MESQUITA, M. O. et al. "Microbiological quality in the roast chicken process in nutritional and nourishment unit. "Food Science and Technology. Campinas, v. 26, n1, p. 198-203, jan-mar. 2006.

NADVORNY, A.; FIGUEIREDO, D. M.; SCHMIDT, V. Ocorrência de Salmonella sp. em surtos de doenças transmitidas por alimentos no Rio Grande do Sul em 2000. Acta Scientiae Veterinariae. Porto Alegre, v. 32, n 1, p. 47-51, abr-mai 2004. https://doi.org/10. 22456/1679-9216.16802

NAUTA, M. J. et al. Food safety in the domestic environment: the effect of consumer risk information on human disease risks. Risk Analysis, v. 28, n 1, p. 179-192, mar 2008. https://doi.org/10.1111/i.1539-6924.2008.01012.x

OLIVEIRA, D. M.; RESENDE, P. O. Fasciola hepatica: ecologia e trajetória históricogeográfica pelo Brasil. Estação Científica (UNIFAP), v. 7, n 2, p. 09-19, jan. 2017. https://doi.org/10.18468/estcien.2017v7n2.p09-19

PERESI, J. T. M. et al. Surtos de enfermidades transmitidas por alimentos causados por Salmonella enteritidis. Revista de Saúde Pública, São Paulo, vol 32, n 5, p. 477-483, out. 1998. https://doi.org/10.1590/S0034-89101998000500011

REDMOND, E. C.; GRIFFITH, C. J. Consumer food handling in the home: a review of food safety studies. Journal of food protection, Des Moines, vol 66, n 1, p. 130-161, 2003.

SANTOS, M. H. R. et al. Segurança alimentar na manipulação domé1stica, abordagem física, química e biológica. III Simpósio de Segurança Alimentar, Florianópolis: mai. \jun (2011): 42-47. [Internet]. [citado 19 de setembro de 2016]. Disponível em: http://pg.utfpr. edu.br/dirppg/ppgep/ebook/2010/CONGR ESSOS/SSA/1

SÃO PAULO. Secretaria Estadual de Saúde.
Divisão de doenças de transmissão hídrica e alimentar. "Vigilância Epidemiológica das Doenças Transmitidas por Água e AlimentosInvestigação de surtos" São Paulo, 2008, p. 86.

TONDO, E. C. BARTZ, S. "Microbiologia e Sistemas de Gestão da Segurança de Alimentos". Vol. 2. Porto Alegre: Editora Sulina; 2014.

VAN AMSON, G.; HARACEMIV S. M. C.; MASSON, M. L. Levantamento de dados epidemiológicos relativos a ocorrências/surtos de doenças transmitidas por alimentos (DTAs) no Estado do Paraná-Brasil, no período de 1978 a 2000. Ciênc. Agrotec. Lavras, v.30, n 6, p. 1139-45, nov-dez, 2006. https://doi.org/10.1590/S1413-70542006000600016

VAN ASSELT, E. D. et al. Cross-contamination in the kitchen: estimation of transfer rates for cutting boards, hands and knives. Journal of Applied Microbiology, vol 105, n 5, p. 1392-1401, mar./maio 2008. https://doi.org/ 10.1111/j.1365-2672.2008.03875.x

WELKER, C. A. D. et al. Análise microbiológica dos alimentos envolvidos em surtos de doenças transmitidas por alimentos (DTA) ocorridos no estado do Rio Grande do Sul, Brasil. Rev. Bras. Biociências. Porto Alegre, vol 8, n 1, p.44-8, jan-mar. 2010.

WILCOK, A. et al. Consumer attitudes, knowledge and behaviour: a review of food safety issues. Trends in Food Science \& Technology. vol 15, n 2, p. 56-66, fev. 2004. https://doi.org/10.1016/j.tifs.2003.08.004

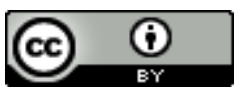

License information: This is an openaccess article distributed under the terms of the Creative Commons Attribution License, which permits unrestricted use, distribution, and reproduction in any medium, provided the original work is properly cited.

Artigo recebido em 10 de outubro de 2018.

Avaliado em 31 de outubro de 2019.

Aceito em 06 de novembro de 2019.

Publicado em 21 de novembro de 2019. 


\section{Como citar este artigo (ABNT):}

SORAGNI, Larissa; BARNABE, Anderson Sena; MELLO, Tatiana Ribeiro de Campos. Doenças transmitidas por alimentos e participação da manipulação inadequada para sua ocorrência: uma revisão. Estação Científica (UNIFAP), Macapá, v. 9, n. 2, p. 19-31, abr./jun. 2019. 